\title{
Sascha Kneip
}

\section{Rolle und Einfluss des Bundesverfassungsgerichts in international vergleichender Perspektive}

Im Jahr 1975 gab das Nachrichtenmagazin »Der Spiegel« einem Beitrag über das Bundesverfassungsgericht den schönen Titel: »Zuchtmeister für Bonn und Bürger «. ${ }^{1}$ Anlass des Artikels war das umstrittene Urteil des Gerichts zum Schwangerschaftsabbruch, das den damals weit verbreiteten Eindruck verstärkte, das Bundesverfassungsgericht - oder zumindest sein Erster Senat - verfolge eine eigene politische Agenda gegen die regierende sozialliberale Koalition und verhindere gezielt einen politisch gewollten Freiheitszugewinn der (hier: weiblichen) Bevölkerung. ${ }^{2}$ Während die zugeschriebene 'Zuchtmeisterrolle< gegenüber der Bevölkerung heute anachronistisch anmutet - das Bundesverfassungsgericht ist in der öffentlichen Wahrnehmung nichts weniger als »das Bürgergericht ${ }^{3}$ der Bundesrepublik Deutschland -, klingt die Klage, das Gericht mache mitunter >Politik < gegen die jeweils Regierenden, weitaus vertrauter. Sie geht meist einher mit spezifischen Rollenzuschreibungen an das Bundesverfassungsgericht, das mal als »Nebenregierung ${ }^{4}{ }^{4}$ mal als »Ersatzgesetzgeber $\ll,{ }^{5} »$ dritte Parlamentskammer ${ }^{6}{ }^{6} »$ Vetospieler «, 7 oder » Reparaturbetrieb des Parlamentarismus « ${ }^{8}$ gesehen wird. Dem ehemaligen Kanzleramtsminister Horst Ehmke wird angesichts des im Jahr 1973 in Karlsruhe anhängigen Verfahrens zum Grundlagenvertrag mit der DDR sogar der Satz zugeschrieben, man lasse sich von den »acht Arschlöchern in Karlsruhe die neue Ostpolitik nicht kaputtmachen«. Auch wenn dies keine soziologische Rollenzuschreibung im engeren Sinne gewesen sein mag, wird doch deutlich, dass Ehmke im Bundesverfassungsgericht eher

1 Der Spiegel 10/1975 vom 3.3.1975, S. 62-67.

2 Vgl. zu dieser Einschätzung auch Uwe Wesel, Der Gang nach Karlsrube. Das Bundesverfassungsgericht in der Geschichte der Bundesrepublik, München 2004, S. $247 \mathrm{ff}$.

3 Vgl. z.B. Jutta Limbach, Das Bundesverfassungsgericht, München 2001, S. $7 \mathrm{ff}$.

4 Vgl. Manfred G. Schmidt, Regieren in der Bundesrepublik Deutschland, Opladen 1992, S. 46.

5 Vgl. Rupert Scholz, »Das Bundesverfassungsgericht: Hüter der Verfassung oder Ersatzgesetzgeber? « in: Aus Politik und Zeitgeschichte 49, Nr. B16 (1999), S. 3-8; Georg Blasberg, Verfassungsgerichte als Ersatzgesetzgeber. Entscheidungsaussprüche bei Normenkontrollen von Bundesverfassungsgericht und Corte Costituzionale, Frankfurt a. M. 2003.

6 Vgl. Alec Stone, The Birth of Judicial Politics in France. The Constitutional Council in Comparative Perspective, Oxford 1992.

7 Vgl. Uwe Wagschal, »Verfassungsgerichte als Vetospieler in der Steuerpolitik « in: Michael Becker / Ruth Zimmerling (Hg.), Politik und Recht. PVS-Sonderheft 36/2006, S. 559-584.

8 Vgl. Hans-Peter Schneider, »Acht an der Macht! Das Bundesverfassungsgericht als >Reparaturbetrieb< des Parlamentarismus? « in: Neue Juristische Wochenschrift 52, Nr.18 (1999), S. 1303-1305. 
eine oppositionelle Neben- oder Gegenregierung als einen unterstützenden Reparaturbetrieb sah.

Die Klage, dass das Bundesverfassungsgericht seine Rolle gegenüber der Politik zu sehr ausdehne und sein Einfluss vielleicht zu groß sei, zieht sich durch die gesamte sechzigjährige Geschichte des Gerichts. Gerade bei umstrittenen oder folgenreichen Urteilen aus Karlsruhe wurde und wird sowohl in der Politik, aber auch in der Wissenschaft und der Öffentlichkeit gerne die Frage gestellt, ob die Machtfülle und der Einfluss des Gerichts nicht vielleicht doch zu groß seien. Gerade im internationalen Vergleich, so liest und hört man mitunter, gebe es doch deutlich weniger machtvolle - und vor allem: zurückhaltendere - Gerichte, die der Demokratie vielleicht besser zu Gesicht stünden, indem sie nicht so stark und so häufig in die Politik shineinregierten< wie das Bundesverfassungsgericht.

Diese Hypothese soll im Folgenden anhand zweier zusammenhängender Fragen aufgegriffen und diskutiert werden. Die erste Frage ist empirischer Natur. Es ist zunächst danach zu fragen, ob das Bundesverfassungsgericht im internationalen Vergleich tatsächlich ein so starkes Gericht ist, wie häufig behauptet wird, und, wenn ja, woran sich dies empirisch festmachen lässt. Damit zusammenhängend soll hier auch der Frage nachgegangen werden, ob Karlsruhe tatsächlich empirisch betrachtet als >Vetospieler oder >Gegenregierung < bezeichnet werden kann - letztlich der Frage also, wie häufig das Bundesverfassungsgericht tatsächlich in >die Politik< interveniert.

Der zweite, damit verknüpfte Fragenkomplex ist ein eher normativer. Hier soll danach gefragt werden, was aus Sicht der Demokratietheorie die Rolle des Bundesverfassungsgerichts im demokratischen Prozess ist bzw. sein sollte und welchen Rollentypus das Gericht eigentlich verkörpert. Ist es ein >Hüter der Demokratie und ihrer Verfassung oder eher ihr >Präzeptor<? Ist der Einfluss, den es auf die Politik ausübt, ein für die Demokratie positiver? Und unterscheidet es sich hier von anderen Verfassungsgerichten der Welt? Diesen Fragen wird im zweiten Teil der vorliegenden Ausführungen nachgegangen.

\section{Die Stärke des Bundesverfassungsgerichts - einzigartig im internationalen Vergleich?}

Zunächst zur ersten - empirischen - Frage: Wie machtvoll und einflussreich ist das Bundesverfassungsgericht eigentlich tatsächlich im internationalen Vergleich? Und: Ist es wirklich eine >Gegenregierung $<$ im politischen System der Bundesrepublik?

Will man das Bundesverfassungsgericht mit anderen höchsten Gerichten bzw. Verfassungsgerichten vergleichen, braucht man Vergleichsmaßstäbe. Im Falle der Verfassungsgerichtsbarkeit - und für die hier zu behandelnde spezifische Frage - bieten sich drei Kriterien an: erstens die formale und faktische Machtfülle des Gerichts, zweitens seine institutionelle Unabhängigkeit von der Politik und drittens seine faktische Interventionstätigkeit. Tatsächlich ist das Bundesverfassungsgericht hinsichtlich aller drei Maßstäbe im internationalen Vergleich herausragend, aber keineswegs einzigartig. 


\section{1 Formale und faktische Machtfülle}

Die formale Machtfülle eines Verfassungsgerichts wird in der empirischen Verfassungsgerichtsforschung meist anhand der formalen Kompetenzen und der Offenheit des Gerichtszugangs gemessen. ${ }^{9}$ Hinsichtlich seiner formalen Kompetenzen verfügt das Bundesverfassungsgericht über nahezu alle zentralen verfassungsgerichtlichen Zuständigkeiten und Befugnisse. Es besitzt neben den bekannten Kompetenzen in konkreten und abstrakten Normenkontrollverfahren, Verfassungsbeschwerden und horizontalen wie vertikalen Kompetenzstreitverfahren auch Kompetenzen im Bereich des objektiven Verfassungsschutzes (z. B. die Entscheidungsbefugnis über Parteiverbote, die Wahlgerichtsbarkeit oder Amtsenthebungsverfahren) sowie die Kompetenz, überstaatliche Normen und das Völkerrecht an der Verfassung zu messen. ${ }^{10}$ Diese formale Machtfülle ist zwar durchaus herausragend, aber keineswegs einzigartig in der Welt. Viele der neu geschaffenen Verfassungsgerichte Süd- und Osteuropas beispielsweise weisen sehr ähnliche Kompetenzstrukturen auf (z. B. die Verfassungsgerichte Spaniens und Portugals, Ungarns, ${ }^{11}$ Tschechiens, der Slowakei, Sloweniens oder Polens), und auch in alten westlichen Demokratien (Österreich, Belgien), Lateinamerika und Asien lassen sich Gerichte finden, die ähnlich viele Kompetenzen auf sich vereinen. ${ }^{12}$

Hinsichtlich des Gerichtszugangs gehört das Bundesverfassungsgericht zu den im internationalen Vergleich >offensten< Gerichten, besitzt aber auch hier kein Alleinstellungsmerkmal. Die Offenheit des Gerichtszugangs zum Bundesverfassungsgericht rührt von der vergleichsweise großen Anzahl klagebefugter Akteure in Verbindung mit relativ geringen faktischen Antragshürden her. Zu den potentiellen Antragstellern gehören, je nach Verfahrensweg, unter anderem die Exekutiven von Bund und Ländern, der Bundestag (bzw. ein Viertel seiner Mitglieder), Gerichte, Parteien und Verbände bis hin zu jedem einzelnen Bürger. Während die Exekutiven in nahezu allen Vergleichsfällen vor den jeweiligen Verfassungsgerichten antragsberechtigt sind, unterscheiden sich die Hürden für Verfassungsbeschwerden durch die Legislative schon deutlicher, wenn auch letztlich nur moderat. Ist die Legislative überhaupt antragsberechtigt (dies ist regelmäßig

9 Vgl. hierzu ausführlich Sascha Kneip, Verfassungsgerichte als demokratische Akteure. Der Beitrag des Bundesverfassungsgerichts zur Qualität der bundesdeutschen Demokratie, Baden-Baden 2009, S. 158ff.; siehe auch Tom Ginsburg, Judicial Review in New Democracies. Constitutional Courts in Asian Cases, Cambridge 2003, S.34ff. und Christoph Hönnige, Verfassungsgericht, Regierung und Opposition. Die vergleichende Analyse eines Spannungsdreiecks, Wiesbaden 2007, S. $101 \mathrm{ff}$.

10 Vgl. hierzu auch die rechtsvergleichende Studie von Alexander von Brünneck, Verfassungsgerichtsbarkeit in den westlichen Demokratien, Baden-Baden 1992.

11 Die jüngsten Verfassungsänderungen in Ungarn haben das dortige Gericht zweifellos geschwächt; es gehört aber nach wie vor zu den formal stärksten Gerichten im EU-27-Raum. Vgl. Kriszta Kovács / Gábor Attila Tóth, »Hungary’s Constitutional Transformation « in: European Constitutional Law Review 7, Nr. 2 (2011), S. 183-203.

12 Vgl. Norbert Lösing, Die Verfassungsgerichtsbarkeit in Lateinamerika, Baden-Baden 2001; Sascha Kneip, »Verfassungsgerichtsbarkeit im Vergleich « in: Oskar W. Gabriel / Sabine Kropp (Hg.), Die EU-Staaten im Vergleich, Wiesbaden 2008, S. 631-655; Tom Ginsburg, Judicial Review in New Democracies, aaO. (FN 9). 
nur bei spezialisierten Verfassungsgerichten der Fall, die abstrakte Normenkontrollverfahren kennen), ${ }^{13}$ liegt das benötigte Quorum in aller Regel zwischen 10 Prozent und einem Drittel der gewählten Parlamentarier. Die Bundesrepublik liegt hier mit ihrem 25Prozent-Quorum im oberen mittleren Bereich, lediglich Belgien sticht mit einem benötigten Quorum von zwei Dritteln der Parlamentarier deutlich heraus.

Am wichtigsten für die Offenheit des Gerichtszugangs (und auch die faktische Wirkmächtigkeit eines Verfassungsgerichts) ist das Institut der Verfassungsbeschwerde, und hier insbesondere jenes der Urteilsverfassungsbeschwerde. Verfassungsbeschwerde und Urteilsverfassungsbeschwerde kennen bei Weitem nicht alle spezialisierten Verfassungsgerichte, in Europa zum Beispiel nur die Gerichte in Belgien, Deutschland, Estland, der Slowakei, Slowenien, Spanien und Tschechien. Österreich und Polen haben zwar generell Verfassungsbeschwerden institutionalisiert, nicht aber solche gegen Gerichtsentscheidungen. Hinzu kommt, dass nicht in allen Fällen mit einer Verfassungsbeschwerde auch Erga-omnes-Folgen eintreten, das Urteil des Verfassungsgerichts also nicht nur für den konkret verhandelten Fall gilt, sondern für alle anderen vergleichbaren Fälle. In Ungarn kann zudem nicht direkt gegen Grundrechtsverstöße an sich geklagt werden, sondern nur gegen die zugrunde liegenden Rechtsnormen. Im Lichte dieser unterschiedlichen institutionellen Ausgestaltungen der Verfassungsbeschwerde erscheint die bundesdeutsche Regelung vergleichsweise weitreichend. Die Antragshürden sind - ungeachtet der hohen Nichtannahmequote - formal nicht allzu hoch, zugleich sind die Folgen eines Urteilsspruchs gravierend (d. h. bindend für die gesamte Staatsgewalt sowie private Akteure), und neben Gesetzesnormen sind auch Handlungen der Exekutive und der Gerichte per Verfassungsbeschwerde angreifbar. Die Verfassungsbeschwerde lässt in der Bundesrepublik prinzipiell jeden Bürger zum potentiellen Antragsteller werden (solange er nachweisen kann, gegenwärtig und unmittelbar in seinen Grundrechten verletzt zu sein), was nicht nur die Macht der Bürger selbst, sondern auch jene des Gerichts stärkt, da es potentiell häufiger in verfassungsrechtliche Streitigkeiten eingeschaltet werden kann. Die Urteilsverfassungsbeschwerde gegen Gerichtsentscheide führt darüber hinaus dazu, dass betroffene Bürger nicht nur gegen Gesetze, sondern auch gegen exekutives und richterliches Handeln klagebefugt sind, was eine deutliche Ausdehnung des Grundrechtsschutzes zur Folge hat. Die konkrete Ausgestaltung der Verfassungsbeschwerde in der Bundesrepublik hebt das Bundesverfassungsgericht also durchaus etwas aus der Menge der Verfassungsgerichte heraus.

Gleichwohl darf man konstatieren, dass die formale Machtfülle des Gerichts insgesamt zwar groß, aber durchaus nicht einzigartig ist. Trügt also der weit verbreitete Eindruck, dass das Gericht mächtiger ist als andere Verfassungsgerichte der Welt, den US-amerikanischen Supreme Court vielleicht ausgenommen?

13 Auch spezialisierte Verfassungsgerichte gewähren der Legislative (oder legislativen Minderheiten) nicht immer ein Antragsrecht. Insbesondere in den Fällen, in denen eine Überprüfung von Normen auf deren Verfassungsmäßigkeit nur inzident erfolgt (also nur innerhalb eines konkreten Rechtsstreits), bleibt die Legislative häufig außen vor. Dies ist beispielsweise in Italien der Fall. 
Der Eindruck trügt nicht. Die formale Machtfülle des Bundesverfassungsgerichts wird durch einen entscheidenden Faktor in tatsächliche Macht umgewandelt, von dem andere Gerichte nicht ohne Weiteres Gebrauch machen können: eine extrem hohe empirische Legitimität in der Bevölkerung. Verglichen mit anderen Verfassungsgerichten und obersten Gerichtshöfen der Welt verfügt das Bundesverfassungsgericht gegenüber den im engeren Sinne politischen Akteuren (etwa der Regierung, dem Parlament und den Parteien) über deutlich höhere Zustimmungswerte, was es diesen anderen Akteuren nahezu unmöglich macht, Urteile des Verfassungsgerichts offen zu ignorieren oder auch nur heimlich nicht umzusetzen (obwohl dies natürlich hin und wieder versucht wird und mitunter auch gelingt). ${ }^{14}$ Die vom Institut für Demoskopie in Allensbach 2012 gemessenen 75 Prozent Zustimmung zu seiner Arbeit ${ }^{15}$ mögen auch der hitzigen Diskussion um die Euro-Rettung und den hohen Erwartungen an das Gericht in diesem Zusammenhang geschuldet gewesen sein. Es lässt sich aber schon seit Beginn der 1980er Jahre feststellen, dass sich die empirische Legitimität des Bundesverfassungsgerichts verfestigt hat, während jene der im engeren Sinne politischen Organe kontinuierlich gesunken ist. Die Vertrauensraten in Bezug auf die Arbeit des Verfassungsgerichts liegen seit Anfang der 1980er Jahre kontinuierlich zwischen 60 und 70 Prozent; in Bezug auf die Arbeit der Bundesregierung haben sie sich im gleichen Zeitraum von 50 auf 25-30 Prozent fast halbiert, für die Parteien liegen sie noch deutlich darunter. ${ }^{16}$ In dieser Konstellation können es sich die politischen Akteure in der Bundesrepublik in der Regel nicht leisten, Urteile aus Karlsruhe offen zu missachten. Die hohe Zustimmung zum Gericht und seiner Arbeit lässt also aus formaler Machtfülle tatsächliche entstehen.

Die hohe empirische Legitimität des Bundesverfassungsgerichts ist auch im internationalen Vergleich durchaus außergewöhnlich. Weder die Verfassungsgerichte Mittel-, Süd- und Osteuropas noch jene in Lateinamerika, Asien oder Afrika können, von wenigen Ausnahmen abgesehen, mit ähnlich hohen Vertrauens- und Zustimmungswerten aufwarten. In Europa kommen allenfalls der österreichische Verfassungsgerichtshof und das polnische Verfassungstribunal an die hohen Werte des deutschen Gerichts heran. In Lateinamerika hingegen ist das Vertrauen in höchste Gerichte meist nur sehr schwach ausgeprägt: Hier ist die gemessene empirische Legitimität der Exekutive häufig doppelt so hoch wie jene der obersten Gerichte oder Verfassungsgerichte, obwohl gerade dort in den vergangenen Jahrzehnten eine deutliche formale Aufwertung der Verfassungsge-

14 Vgl. zu den Umständen der Umsetzung oder Nichtumsetzung von Urteilen des Bundesverfassungsgerichts z. B. Georg Vanberg, The Politics of Constitutional Review in Germany, Cambridge 2005.

15 Vgl. Renate Köcher, »Das Bollwerk « in: Frankfurter Allgemeine Zeitung vom 21.8.2012.

16 Vgl. auch die Daten in Sascha Kneip, Verfassungsgerichte als demokratische Akteure, aaO. (FN 9), S. 198ff.; Georg Vanberg, The Politics of Constitutional Review in Germany, aaO. (FN 14), S. 98; Hans Vorländer / André Brodocz, »Das Vertrauen in das Bundesverfassungsgericht. Ergebnisse einer repräsentativen Bevölkerungsumfrage « in: Hans Vorländer (Hg.), Die Deutungsmacht der Verfassungsgerichtsbarkeit, Wiesbaden 2006, S. 259-295. 
richtsbarkeit stattgefunden hat. ${ }^{17}$ Auch das Ansehen des US-amerikanischen Supreme Courts hat in den letzten Jahren stark gelitten, wie aktuelle Untersuchungen aus den USA zeigen; ${ }^{18}$ das US-Gericht wird offenbar mehr und mehr als ein politischer Akteur wahrgenommen, der seine Urteile weniger auf Grundlage einer neutralen Auslegung der Verfassung fällt als aufgrund persönlicher richterlicher Präferenzen. ${ }^{19}$ Dem Bundesverfassungsgericht scheint es sehr viel besser als anderen Gerichten gelungen zu sein, die Bürgerinnen und Bürger von der Qualität seiner Arbeit zu überzeugen.

\section{2 Institutionelle Unabhängigkeit}

Ein wesentlicher Grund für das Vertrauen der Bürger in das Bundesverfassungsgericht dürfte in seiner institutionellen Unabhängigkeit liegen. Die institutionelle Unabhängigkeit eines Gerichts wird meist anhand der Inklusivität des Richterwahlverfahrens und der anschließenden Unabhängigkeit der Richterinnen und Richter von politischem Druck gemessen, also vor allem anhand der Amtszeiten der Richterinnen und Richter und möglicher Wieder- und Abwahlmöglichkeiten. ${ }^{20}$ Auch hier steht das Bundesverfassungsgericht im internationalen Vergleich überaus gut da. In Europa sind nur noch die Gerichte in Lettland, Litauen, Belgien und der Slowakei in ähnlich hohem Ausmaß formal unabhängig, ${ }^{21}$ in Lateinamerika nur das oberste Gericht Argentiniens und mit Einschränkungen jene in Brasilien und Mexiko. Unter den asiatischen Verfassungsgerichten kann nur das südkoreanische Verfassungsgericht als zumindest ähnlich unabhängig gelten. ${ }^{22}$

Entscheidend für die hohe institutionelle Unabhängigkeit des Bundesverfassungsgerichts sind vor allem drei Faktoren: das Richterwahlverfahren, die Regelungen zur Amtszeit der Richterinnen und Richter und die mit der Statusdenkschrift von 1952 selbst etablierte Stellung als gleichberechtigtes Verfassungsorgan der Bundesrepublik Deutschland. Das Richterwahlverfahren ist immer wieder - und durchaus zu Recht - in die Kritik

17 Vgl. die Daten des Latinobarometro (www.latinobarometro.org) und Gretchen Helmke / Julio Ríos-Figueroa, »Introduction: Courts in Latin America « in: dies. (Hg.), Courts in Latin America, Cambridge 2011, S. $3 \mathrm{ff}$.

18 Vgl. die entsprechenden Daten des Gallup-Instituts unter http://www.gallup.com/poll/4732/ supreme-court.aspx (Zugriff am 30.11.2012). Sie zeigen, dass die Zustimmungsraten zur Arbeit des Supreme Courts von noch 62 Prozent im Jahr 2000 auf 46 Prozent im Spätsommer 2012 gesunken sind.

19 Allerdings sei generell darauf hingewiesen, dass es derzeit keine aktuellen Daten aus vergleichend angelegten Erhebungen zum Vertrauen in Verfassungsgerichte bzw. höchste Gerichte gibt. Die in diesem Papier angeführten Daten stammen jeweils aus Einzelerhebungen und sind insofern in vergleichender Hinsicht mit Vorsicht zu genießen. Vgl. aber James L. Gibson / Gregory A. Caldeira / Vanessa A. Baird, »On the Legitimacy of High Courts” in: American Political Science Review 92, Nr. 2 (1998), S. 343-358.

20 Vgl. auch Tom Ginsburg, Judicial Review in New Democracies, aaO. (FN 9), S. 42ff.

21 Vgl. Sascha Kneip, »Verfassungsgerichtsbarkeit im Vergleich«, aaO. (FN 12); Julio RíosFigueroa, »Institutions for Constitutional Justice in Latin America in: Gretchen Helmke / Julio Ríos-Figueroa (Hg.), Courts in Latin America, aaO. (FN 17), S. $28 \mathrm{ff.}$

22 Vgl. Tom Ginsburg, Judicial Review in New Democracies, aaO. (FN 9), S. $242 \mathrm{ff}$.

ZfP 60. Jg. 1/2013 
geraten. Insbesondere die Rolle des Richterwahlausschusses des Bundestages und seine intransparente Art der Kandidatenkür entsprechen, trotz anderweitiger Einschätzungen des Bundesverfassungsgerichts selbst, ${ }^{23}$ wohl kaum den Vorgaben des Grundgesetzes. ${ }^{24}$ Statt einen geheim tagenden Ausschuss mit der Richterwahl zu betrauen, wäre es demokratietheoretisch wie verfassungsrechtlich sauberer, das Bundestagsplenum selbst mit der Wahl der Richterinnen und Richter zu befassen. Dies gilt umso mehr, als der große Erfolg der Richterwahlen in der Bundesrepublik nicht auf ihrem intransparenten Verfahren beruht als vielmehr auf dem die Inklusivität fördernden Zwei-Drittel-Quorum für die Wahl der Richterinnen und Richter. Dieses - und nicht das Aussuchen der Kandidatinnen und Kandidaten hinter verschlossenen Türen - ist der Garant für die Güte und Unabhängigkeit der Richterinnen und Richter am Bundesverfassungsgericht. Die beiden großen Volksparteien CDU/CSU und SPD sind auf diese Weise gezwungen, sich auf konsensfähige Kandidaten zu einigen - und haben so in der Vergangenheit nahezu ausnahmslos honorige und fachlich über jeden Zweifel erhabene Richterinnen und Richter in Karlsruhe installiert.

Dieses konsensdemokratische Moment des bundesdeutschen Richterwahlverfahrens ist im Rest der Welt alles andere als weit verbreitet. In Europa verlangen nur noch Belgien, Portugal und Ungarn sowie - bei einem Teil der zu bestellenden Richter - Spanien und Italien übergroße Mehrheiten bei der Richterwahl; häufig reicht eine einfache Mehrheit im Parlament zu deren Bestellung. Gerade in präsidentiellen oder semipräsidentiellen Regierungssystemen erfolgt die Wahl häufig mit einfacher Mehrheit auf Vorschlag des Staatspräsidenten; mitunter benennt der Präsident die Richterinnen und Richter gleich selbst.

Auch die konkrete Ausgestaltung der Amtszeiten macht die Richterinnen und Richter des Bundesverfassungsgerichts vergleichsweise unabhängig von der Politik. Seit der Abschaffung der Wiederwahlmöglichkeit im Jahr 1970 ist dafür gesorgt, dass die Richter unbeeinflusst von möglichen Wiederwahlerfordernissen ihrer Arbeit nachgehen können. Die zwölfjährige Amtszeit ist zudem hinreichend lange ausgestaltet; die zeitliche Begrenzung trägt im Vergleich zu - im Grunde demokratiefremden - Amtszeiten auf Lebenszeit dazu bei, keine gottgleichen Richterpersönlichkeiten hervorzubringen oder vorauszusetzen..$^{25}$ Eine Abwahl der Richterinnen und Richter ist im Falle des Bundesverfassungsgerichts nicht möglich, was ebenfalls ihre Unabhängigkeit von der Politik deutlich stärkt. Die Unmöglichkeit der Abwahl von Verfassungsrichtern ist im internationalen Vergleich allerdings mittlerweile üblich. In Europa ist eine Abwahl höchster

23 Vgl. BVerfG, 2 BvC 2/10 vom 19.6.2012.

24 Siehe z. B. die eindeutige Einschätzung bei Hans D. Jarass / Bodo Pieroth, Grundgesetz für die Bundesrepublik Deutschland. Kommentar, 10. Auflage, München 2009, S. 966 (mit weiteren Nachweisen).

25 Gleichwohl werden noch immer in vielen Fällen oberste Richter auf Lebenszeit gewählt - in Europa immerhin in 13 der 27 EU-Mitgliedsstaaten. Mitunter wird eine Pensionsgrenze definiert, die dann in der Regel zwischen 65 und 70 Jahren liegt. Zeitliche Begrenzungen der Amtszeiten oberster Richter sind auch außerhalb Europas eher die Ausnahme als die Regel. 
Richter nur noch in Irland, Großbritannien und Malta grundsätzlich vorgesehen. ${ }^{26}$ In den anderen Fällen können allenfalls die Gerichte selbst unter bestimmten Umständen etwa bei einer strafrechtlichen Verfolgung amtierender Richter - über eine Abberufung befinden.

Eine Unabhängigkeitserklärung besonderer Art stellt die Statusdenkschrift des Bundesverfassungsgerichts von 1952 dar, mit der sich das Gericht selbst zum gleichberechtigten Verfassungsorgan erklärte und erfolgreich seine "Ressortierung « beim Justizministerium rückgängig machte. ${ }^{27}$ Für die Unabhängigkeit des Bundesverfassungsgerichts hat der Status als Verfassungsorgan - jenseits der nicht unwichtigen politischen Symbolik - nicht zu überschätzende Folgen: Als selbstständiges Verfassungsorgan verfügt das Gericht über einen eigenen Etat im Haushaltsgesetz (und ist nicht dem Etat des Justizministeriums zugeordnet), es ist keiner ministeriellen Dienstaufsicht unterworfen, es kann in eigener Verantwortung Mitarbeiterinnen und Mitarbeiter auswählen und begegnet damit den anderen Verfassungsorganen auf Augenhöhe. Es kann mit anderen Worten nicht über dienstrechtliche oder finanzielle Anreize und Sanktionen politisch diszipliniert werden. Zusammen mit der konsensorientierten Richterauswahl und den institutionellen Regelungen zur Amtszeit der Richter stellt dies - in Verbindung mit dem oben thematisierten Legitimationspuffer in der Bevölkerung - sicher, dass das Bundesverfassungsgericht auch faktisch überaus unabhängig agieren kann. »Über dem Verfassungsgericht«, so schon der durch das Gleichberechtigungsstreben des Gerichts enervierte Justizminister Thomas Dehler im Jahr 1952, »steht nur noch der liebe Gott « ${ }^{28}$ - und auch der mischt sich in der Regel nicht in die Karlsruher Entscheidungsfindung ein.

\section{3 Faktische Interventionstätigkeit}

Wie steht es nun - drittens - mit der faktischen Interventionstätigkeit des Bundesverfassungsgerichts? Resultiert aus der formalen und faktischen Machtfülle und Unabhängigkeit des Gerichts eine entsprechende Nutzung dieser institutionellen Stärke? Regiert das Gericht also tatsächlich häufig in >die Politik hinein? Und unterscheidet es sich hier deutlich von anderen Verfassungsgerichten? Die Antwort lautet gut sozialwissenschaftlich: ja und nein.

Zunächst einmal stimmt es, dass das Bundesverfassungsgericht mit immer mehr Klagen befasst wird und über die Jahre und Jahrzehnte alleine dadurch schon zu einem stärkeren potentiellen Mitspieler in der bundesdeutschen Politik geworden ist. Bis Ende der 1950er Jahre erreichten weniger als 1.000 Verfahren pro Jahr das Karlsruher Gericht.

26 In Tschechien können Richter nach rechtskräftiger Verurteilung aus dem Amt entlassen werden; Gleiches gilt in Slowenien, wo das Parlament Richter auch auf Antrag des Richterrates abberufen kann.

27 Vgl. Uwe Wesel, Der Gang nach Karlsrube, aaO. (FN 2), S. 76ff.; Oliver Lembcke, Hüter der Verfassung. Eine institutionentheoretische Studie zur Autorität des Bundesverfassungsgerichts, Tübingen 2007, S. 73ff.

28 Zitiert in Uwe Wesel, Der Gang nach Karlsrube, aaO. (FN 2), S. 81.

ZfP 60. Jg. 1/2013 
Mittlerweile sind es regelmäßig zwischen 6.000 und 6.500 Klagen pro Jahr. ${ }^{29}$ Das sind annähernd so viele Klagen, wie sie den US Supreme Court pro Jahr erreichen, der aber anders als das Bundesverfassungsgericht frei über die Annahme von Klagen entscheiden kann und nur über etwa 80 Klagen pro Jahr tatsächlich befindet. ${ }^{30}$ Allerdings weist beispielsweise das spanische Verfassungsgericht etwa 7.000 Verfahrenseingänge pro Jahr aus ${ }^{31}$ der österreichische Verfassungsgerichtshof je nach Jahr zwischen 3.000 und 6.000 Verfahren (2011: 4.400 neu anhängig gewordene Verfahren). ${ }^{32}$ Das slowenische Verfassungsgericht erhält und entscheidet jährlich etwa 1.800 Verfahren. ${ }^{33}$ Niedriger sind die Verfahrenszahlen beispielsweise in Ungarn (1.000 bis 2.000 neue Verfahren pro Jahr), ${ }^{34}$ Portugal (hier fallen durchschnittlich etwa 1.200 Klagen im Jahr an) ${ }^{35}$ oder Polen, wo das Verfassungstribunal pro Jahr nur zwischen 100 und 150 Fälle entscheidet. ${ }^{36}$

Das Bundesverfassungsgericht wird also vergleichsweise häufig in verfassungsrechtliche Streitigkeiten involviert, dies gilt aber ebenso für andere Verfassungsgerichte - nicht zuletzt in Bezug auf die Bevölkerungszahl des jeweiligen Landes. Gerichte, die Verfassungsbeschwerden der Bürger zulassen, sind üblicherweise mit deutlich mehr Klagen konfrontiert als jene Gerichte, die ausschließlich über konkrete oder abstrakte Normenkontrollklagen entscheiden. ${ }^{37}$ Der bekannte Satz Rainer Wahls, dass erfolgreiche Rechtsinstitutionen und -akteure ihre eigene Nachfrage schaffen, gilt, so scheint es, für eine ganze Reihe etablierter Verfassungsgerichte. ${ }^{38}$

29 Vgl. die entsprechenden Jahresstatistiken des Bundesverfassungsgerichts unter http:// www.bverfg.de/organisation.html (Zugriff am 4.12.2012).

30 Den US Supreme Court erreichen pro Jahr knapp 8.000 Klagen; tatsächlich verhandelt wird damit nur über etwa ein Prozent aller Klagen. Vgl. http://www.supremecourt.gov/publicinfo/ year-end/2011year-endreport.pdf (Zugriff am 4.12.2012) und generell zum Annahmeverfahren des US-Supreme-Courts Winfried Brugger, Grundrechte und Verfassungsgerichtsbarkeit in den Vereinigten Staaten von Amerika, Tübingen 1987, S. 9-21.

31 Vgl. http://www.tribunalconstitucional.es/en/tribunal/estadisticas/pages/estadisticas2011.aspx\#A1 (Zugriff am 4.12.2012).

32 Der österreichische Verfassungsgerichtshof ist allerdings auch Revisionsgericht in Asylfragen, was die Verfahrenszahlen mitunter in die Höhe treibt. Vgl. zu den Verfahrenszahlen den Tätigkeitsbericht des Gerichts unter http://www.vfgh.gv.at/cms/vfgh-site/attachments/4/7/5/ CH0011/CMS1334821579438/taetigkeitsbericht_vfgh_2011.pdf (Zugriff am 4.12.2012).

33 Zwischen 2006 und 2008 lagen die Verfahrenszahlen sogar noch deutlich höher. Vgl. für 2011 http://www.us-rs.si/media/annual.report.2011.pdf (Zugriff am 4.12.2012).

34 Vgl. http://www.mkab.hu/letoltesek/statisztika_ossz.pdf (Zugriff am 4.12.2012).

35 Vgl. http://www.tribunalconstitucional.pt/tc/tribunal-estatisticas.html (Zugriff am 4.12.2012).

36 Vgl. http://www.trybunal.gov.pl/eng/statistics/statistics_file.pdf (Zugriff am 4.12.2012).

37 Der französische Verfassungsrat beispielsweise, der bislang keine Verfassungsbeschwerden verhandelt, entscheidet im Jahr in der Regel »nur « über 50 bis 150 Anträge. Vgl. für eine Übersicht der Verfahrenszahlen seit 1958 http://www.conseil-constitutionnel.fr/conseil-constitutionnel/francais/les-decisions/acces-par-date/decisions-depuis-1959/les-decisions-par-date. 4614.html (Zugriff am 4.12.2012).

38 » Rechtsschutz ist ein Wachstumsgut und funktioniert im Sinne der Angebotsökonomie. Er erzeugt durch seine Existenz Nachfrage und Wachstum «; Rainer Wahl, »Die Reformfrage « in: Peter Badura / Horst Dreier (Hg.), Festschrift 50 Jabre Bundesverfassungsgericht. Band 1: Verfassungsgerichtsbarkeit, Verfassungsprozess, Tübingen, S. 461-491, hier S. 476. 
Wie häufig interveniert das Bundesverfassungsgericht, wenn es von anderen Akteuren angerufen und eingeschaltet wird? Wie häufig erklärt es vor allem Normen des Gesetzgebers oder Handlungen der Exekutive für verfassungswidrig und/oder nichtig? Die Interventionshäufigkeit des Bundesverfassungsgerichts ist, in absoluten Zahlen betrachtet, durchaus beachtlich. Insgesamt hat das Bundesverfassungsgericht zwischen 1951 und 2011640 Normen (Gesetze oder Einzelnormen) des Bundes oder der Länder ganz oder teilweise als verfassungswidrig beanstandet. Dies entspricht im Durchschnitt 10,6 verfassungswidrigen Normen pro Jahr. ${ }^{39}$ Der US-amerikanische Supreme Court hingegen erklärte in den mehr als 200 Jahren seines Bestehens gerade einmal etwa 165 Bundesgesetze für ganz oder teilweise verfassungswidrig. Zusammen mit den annullierten Normen auf Bundesstaats- und der lokalen Ebene kommt der Supreme Court seit dem Jahr 1803 - dem Jahr, als er zum ersten Mal im Fall Marbury v. Madison ein verfassungsrechtliches Prüfungsrecht von Gesetzen für sich beanspruchte - auf etwa 1.500 Annullierungen, was statistisch etwa 7,2 Annullierungen pro Jahr entspricht. Nun lässt sich die Rechtsprechungstätigkeit des 19. Jahrhunderts aber nur bedingt mit jener von heute vergleichen. Zwischen 1990 und 2011 annullierte das höchste US-Gericht im Schnitt etwa 5,1 Normen pro Jahr, das Bundesverfassungsgericht kassierte im gleichen Zeitraum etwa 11,1 Normen pro Jahr. ${ }^{40}$ Das Bundesverfassungsgericht weist also eine deutlich höhere Annullierungsquote auf als sein US-amerikanisches Pendant.

Hieraus zu schließen, das Bundesverfassungsgericht würde im internationalen Vergleich übermäßig häufig in >die Politik < intervenieren, wäre aber voreilig. Der österreichische Verfassungsgerichtshof beispielsweise erklärte im Durchschnitt der letzten elf Jahre 28,3 Normen ganz oder teilweise für verfassungswidrig, mit einem Annullierungsmaximum von mehr als jeweils 40 für verfassungswidrig erklärten Normen in den Jahren 2002 bis 2004. ${ }^{41}$ Damit ist der Verfassungsgerichtshof durchaus >konkurrenzfähig<.

Absolute Verfahrens- und Annullierungszahlen erzählen aber nur einen Teil der Geschichte. Zum einen müssen sie, um ein vollständigeres Bild zu erhalten, mit den jeweils bestätigten Normen innerhalb eines Geschäftsjahres kontrastiert werden. Denn die Annullierungen könnten möglicherweise nur einen Bruchteil einer ansonsten normbestätigenden verfassungsgerichtlichen Tätigkeit widerspiegeln, sodass auch aus hohen Annullierungszahlen alleine noch keine Justizialisierungstendenz abgeleitet werden kann. Betrachtet man stattdessen Annullierungsquoten (oder Annullierungssaldi), ${ }^{42}$ hier vergleichend für das Bundesverfassungsgericht und den österreichischen Verfassungsgerichtshof für die letzten zehn Jahre, zeigen sich interessante Trends. Zum einen annullierte der

39 Vgl. die Jahresstatistik 2011 unter http://www.bverfg.de/organisation/statistik_2011.html (Zugriff am 4.12.2012).

40 Vgl. hierzu die Supreme Court Database unter http://scdb.wustl.edu/index.php (Zugriff am 4.12.2012) und die Jahresstatistiken des Bundesverfassungsgerichts, aaO. (FN 29).

41 Vgl. die Tätigkeitsberichte des Gerichts unter http://www.vfgh.gv.at/cms/vfgh-site/vfgh/taetigkeit.html.

42 Der Annullierungssaldo bildet das Verhältnis von Normbestätigungen zu Normannullierungen ab. Werden mehr Normen pro Jahr bestätigt als annulliert, ist der Annullierungssaldo positiv, werden mehr Normen annulliert als bestätigt, ist er negativ.

ZfP 60. Jg. 1/2013 
Verfassungsgerichtshof nicht nur deutlich mehr Normen pro Jahr als das Bundesverfassungsgericht, er wies bis 2007 auch wesentlich größere negative Annullierungssaldi auf. Seit 2008 sind die Annulierungssaldi des Verfassungsgerichts Österreichs allerdings durchgehend positiv, während sich für das Bundesverfassungsgericht - bezieht man die Zeit vor 2001 mit ein - ein gegenläufiger Trend erkennen lässt. Die Annullierungssaldi sind im bundesdeutschen Fall zwar deutlich moderater, aber dafür seit 2002 konstant negativ, d. h., seit 2002 hat das Bundesverfassungsgericht pro Jahr mehr Normen annulliert als bestätigt. Dies schreibt einen Trend fort, der sich schon seit Ende der 1990er Jahre abzeichnete: Bis Mitte der 1990er Jahre ließen sich für das Bundesverfassungsgericht deutlich positive Annullierungssaldi nachweisen, die dann zurückgingen und sich mittlerweile ins Negative verkehrt haben. ${ }^{43}$ Während der Verfassungsgerichtshof sich also in den letzten Jahren von einer zwischenzeitlich vergleichsweise exzessiven Annullierungspraxis verabschiedet hat, scheint das Bundesverfassungsgericht seit Ende der 1990er Jahre den gegenteiligen Weg zu gehen, wenn auch auf insgesamt niedrigerem Niveau.

Abbildung 1: Annullierungssaldi des Bundesverfassungsgerichts und des Verfassungsgerichtshofs im Vergleich

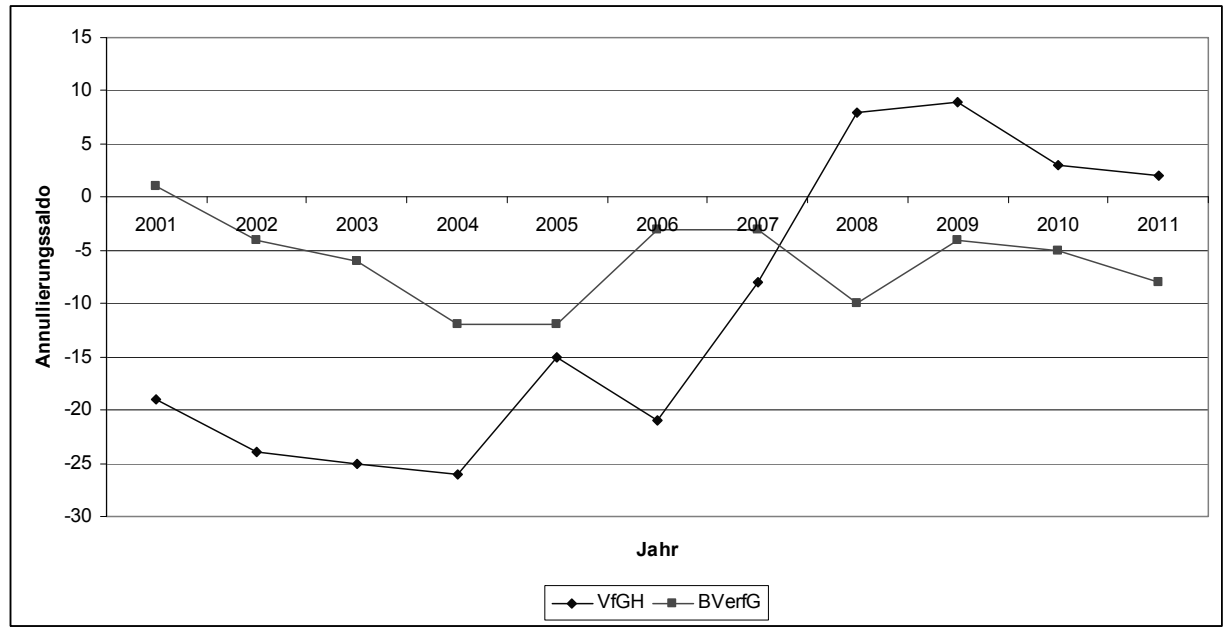

Quelle: eigene Darstellung.

Ein generelles Problem der Aussagefähigkeit quantitativer Annullierungszahlen und -quoten besteht darin, dass ein Spezifikum verfassungsgerichtlichen Urteilens, die verfassungskonforme Auslegung von Normen, mit ihnen nicht abgebildet werden kann. Über die verfassungskonforme Auslegung regieren Verfassungsgerichte auch bei einer Normbestätigung mitunter sehr weitreichend in den Aufgabenbereich des Gesetzgebers

43 Vgl. hierzu ausführlicher Sascha Kneip, »Gegenspieler, Vetospieler oder was? Demokratiefunktionales Agieren des Bundesverfassungsgerichts 1951-2005 in: Politische Vierteljabresschrift 52, Nr. 2 (2011), S. 220-247. 
hinein, indem sie verbindliche Interpretationen des Normtextes vornehmen, die den Gesetzgeber manchmal stärker binden, als wenn die fragliche Norm annulliert und dem Parlament oder der Exekutive zur Neuregelung zurückgegeben würde. Zwar ist die verfassungskonforme Auslegung von ihrem Prinzip her als selbstbeschränkendes Instrument gedacht, das die Einheit der Rechtsordnung sichern und gerade den Vorrang des Gesetzgebers bei der Konkretisierung der Verfassung zum Ausdruck bringen soll. Insbesondere die gesetzgeberischen Grundentscheidungen, Wertungen und Zwecke einer Norm dürfen durch die verfassungskonforme Auslegung prinzipiell nicht verändert werden. ${ }^{44}$ Faktisch wird durch diese Art der Normauslegung der Spielraum der Politik aber zum Teil erheblich begrenzt. ${ }^{45}$

Bezieht man dies in die Analyse ein, lässt sich feststellen, dass das Bundesverfassungsgericht in den letzten zehn bis fünfzehn Jahren stärker in >die Politik< interveniert hat als in den Jahren und Jahrzehnten zuvor; es hat, absolut wie relativ, tatsächlich häufiger Normen des Gesetzgebers beanstandet.

\section{4 Demokratietheoretische Bewertung}

Was heißt dies für die bundesdeutsche Demokratie und die Rolle des Gerichts in ihr? Bedeutet ein häufigeres Hineinregieren in die Politik zugleich, dass das Gericht raus der Rolle gefallen ist und zu Unrecht häufiger als in der Vergangenheit interveniert?

Auch dieser Schluss wäre voreilig. Zum einen hängt die Interventionshäufigkeit eines Verfassungsgerichts idealiter von der Anzahl substantiierter Klagen sowie der verfassungsrechtlichen Güte der Gesetzgebung ab. Eine Zunahme berechtigter Klagen gegen tatsächlich verfassungswidrige Gesetze sollte idealerweise immer zu einer höheren Interventionstätigkeit eines Verfassungsgerichts führen. Zum anderen - und damit zusammenhängend - besteht die Aufgabe eines Verfassungsgerichts aus demokratietheoretischer Sicht gerade darin, diese möglichen Verletzungen der Verfassung und der Kernprinzipien der Demokratie zu prüfen und im Zweifelsfall zu sanktionieren. ${ }^{46}$ Nur dann kommt das Gericht seinem demokratiefunktionalen Auftrag tatsächlich nach. Es fällt damit - bei begründeter Intervention - also keineswegs raus der Rolles, sondern nimmt

44 Vgl. klassisch Konrad Hesse, Grundzüge des Verfassungsrechts der Bundesrepublik Deutschland, Heidelberg 1999, S. 30ff.; siehe zudem die Ausführungen des Bundesverfassungsgerichts selbst zu den Grenzen der verfassungskonformen Auslegung in BVerfGE 54, 277, S. 299 sowie Klaus Schlaich / Stefan Korioth, Das Bundesverfassungsgericht. Stellung, Verfabren, Entscheidungen, 8. Auflage, München 2010, S. 313-314.

45 Siehe für eine kritische Bestandsaufnahme Reinhold Zippelius, »Verfassungskonforme Auslegung von Gesetzen « in: Christian Starck (Hg.), Bundesverfassungsgericht und Grundgesetz. Festgabe aus Anlaß des 25jährigen Bestehens des Bundesverfassungsgerichts. Zweiter Band: Verfassungsauslegung, Tübingen 1976, S. 108-124 und Andreas Voßkuhle, »Theorie und Praxis der verfassungskonformen Auslegung von Gesetzen durch Fachgerichte. Kritische Bestandsaufnahme und Versuch einer Neubestimmung « in: Archiv des öffentlichen Rechts 125, Nr. 2 (2000), S. 177-201.

46 Vgl. ausführlich Sascha Kneip, Verfassungsgerichte als demokratische Akteure, aaO. (FN 9), S. 80-88.

ZfP 60. Jg. 1/2013 
seine Rolle im Gegenteil erst wirklich an. Mit dieser Rollenübernahme ist auch kein Machtverlust der Politik oder deren Justizialisierung verbunden, wie Dieter Grimm schon vor langer Zeit treffend feststellte. Denn idealerweise sorgt das Bundesverfassungsgericht durch seine Interventionen lediglich dafür, dass »sich die übrigen Staatsorgane so verhalten, wie sie sich von Verfassungs wegen ohnedies verhalten müssten «. Der Machtverlust, so Grimm weiter, besteht dann lediglich im »Verlust der Macht, ungestraft gegen die Verfassung zu verstoßen ${ }^{4}{ }^{47}$

Schließt man sich dieser Sichtweise an, eröffnet sich eine interessante demokratietheoretische Perspektive auf das Verhältnis von (Bundes-)Verfassungsgerichtsbarkeit und Politik. Diese sei am Beispiel der Politik der inneren Sicherheit der letzten fünfzehn Jahre kursorisch illustriert: Betrachtet man die Zunahme der Interventionshäufigkeit des Bundesverfassungsgerichts nach Politikfeldern, kann unter anderem für den Bereich der Rechtspolitik eine deutliche Steigerung der Interventionen festgestellt werden. Zurückzuführen ist dies nicht zuletzt auf die Sicherheitspolitik des Bundes und der Länder seit Mitte der 1990er Jahre und vor allem nach dem Jahr 2001. Das Bundesverfassungsgericht hat hier zum Beispiel beanstandet: das sogenannte »Verbrechensbekämpfungsgesetz « ${ }^{48}$ das Gesetz über den »Großen Lauschangriff, « ${ }^{49}$ das Gesetz zum Europäischen Haftbefehl, ${ }^{50}$ das niedersächsische Gesetz über die öffentliche Sicherheit und Ordnung «, ${ }^{11}$ das Luftsicherheitsgesetz, ${ }^{52}$ die Gesetze zur automatischen Kennzeichenerfassung von Hessen und Schleswig-Holstein, ${ }^{53}$ das nordrhein-westfälische Verfassungsschutzgesetz (»Online-Durchsuchung «), ${ }^{54}$ das bayerische Versammlungsgesetz, ${ }^{55}$ das Telekommunikationsgesetz (»Vorratsdatenspeicherung «) ${ }^{56}$ sowie die Gesetze zur Sicherungsverwahrung gefährlicher Straftäter. ${ }^{57}$ In all diesen Fällen beanstandete das Verfassungsgericht unter Beibehaltung seiner tendenziell freiheitserweiternden Rechtsprechung in diesem Politikfeld freiheitsbeschränkende Gesetze des Bundes und der Länder. Die genannten Gesetze sind also weniger an einer neuen Karlsruher Lust zur Intervention gescheitert als mit einer lange gefestigten Rechtsprechung des Bundesverfassungsgerichts kollidiert, was höhere Annullierungszahlen zur logischen Folge werden ließ. Schärfer formuliert: Bundes- wie Landesgesetzgeber haben in den letzten fünfzehn Jahren immer wieder versucht, über (letztlich verfassungswidrige) Sicherheitsgesetze die Freiheitsrechte der Bürgerinnen und Bürger einzuschränken. Indem das Bundesverfassungs-

47 Dieter Grimm, »Verfassungsgerichtsbarkeit - Funktion und Funktionsgrenzen im demokratischen Staat « in: Wolfgang Hoffmann-Riem (Hg.), Sozialwissenschaften im Studium des Rechts. Band 2: Staats- und Verwaltungsrecht, München 1977, S. 83-108, hier S. 87.

48 BVerfGE 100, 313.

49 BVerfGE 109, 279.

50 BVerfGE 113, 273.

51 BVerfGE 113, 348.

52 BVerfGE 115, 118.

53 BVerfGE 120, 378.

54 BVerfGE 120, 274.

55 Einstweilige Anordnung; BVerfGE 122, 342.

56 BVerfGE 125, 260.

57 BVerfGE 128, 326. 
gericht hier intervenierte, hat es durchaus zu einem Machtverlust der gewählten Legislativen und Exekutiven beigetragen. Es hat damit aber, im Grimm'schen Sinne, lediglich die Macht beschränkt, ungestraft gegen die Verfassung zu verstoßen - und damit der bundesdeutschen Demokratie einen großen Dienst erwiesen.

Die genannten Beispiele zeigen, dass der tatsächliche Einfluss des Gerichts auf die Politik nur bedingt quantitativ zu erfassen ist. Hinzutreten müsste jeweils eine Qualifizierung der Karlsruher Rechtsprechung, die etwas genauer erahnen lässt, welche Folgen die Urteilssprüche des höchsten deutschen Gerichts für die Politik im Speziellen und für demokratisches Regieren im Allgemeinen haben. Gemessen an allen Verfahren der letzten fünfzehn Jahre waren die genannten Urteile zu den diversen Sicherheitsgesetzen numerisch unbedeutend; für die Verfassungswirklichkeit der Bundesrepublik aber waren sie von großer Wichtigkeit. ${ }^{58}$

\section{Zur $>$ Rolle des Bundesverfassungsgerichts in der bundesdeutschen Demokratie}

Dies führt uns abschließend zu der Frage, welche Rolle das Bundesverfassungsgericht denn nun eigentlich in der bundesdeutschen Demokratie spielt. Ist sein Agieren der Demokratie zuträglich oder würde weniger verfassungsgerichtliches Agieren vielleicht zu >mehr Demokratie beitragen? Analysiert man die Urteile des Gerichts nach demokratiefunktionalen und -dysfunktionalen Gesichtspunkten, lässt sich ohne Mühe argumentieren, dass das Bundesverfassungsgericht die Qualität der bundesdeutschen Demokratie insgesamt bedeutend gestärkt und erhöht hat. ${ }^{59}$ In der langen, nun schon über sechzigjährigen Geschichte des Gerichts lassen sich nur sehr wenige Urteile finden, von denen sich behaupten ließe, dass sie die bundesdeutsche Verfassung, die Demokratie oder ihre Bürger nicht ausreichend geschützt hätten. $\mathrm{Zu}$ diesen Urteilen gehören sicherlich jene, die in den 1950er, 1960er und 1970er Jahren die Diskriminierung Homosexueller bestätigten, oder jene, die die Anti-Terror-Gesetze der 1970er Jahre allzu bereitwillig billigten. Dazu kann man auch einige richterliche Kompetenzübertretungen in den Bereichen der Sozial-, Steuer- und Finanzpolitik zählen, die den Gesetzgeber hin und wieder seiner eigentlich proklamierten »Einschätzungsprärogative « beraubten. Insgesamt aber lassen sich nur sehr wenige solcher Urteile benennen. Im Gegenteil: Die übergroße Mehrzahl der Karlsruher Urteile dürfen als Meilensteine einer demokratischen und liberalen Verfassungsentwicklung gelten und erscheinen auch im internationalen Vergleich als vorbildlich. Insbesondere die mittlerweile stark ausdifferenzierte Grundrechtsjudikatur des Gerichts hat die demokratische Qualität der Bundesrepublik bedeutend erhöht; es ist

58 Gleiches gilt selbstverständlich auch für andere Politikbereiche wie die Sozial-, Steuer-, Finanzoder Europapolitik. Auch hier zeigt sich der Einfluss des Bundesverfassungsgerichts letztlich eher an konkreten Urteilen als an der Masse der in diesen Bereichen ergangenen Urteile.

59 Ausführlich hierzu Sascha Kneip, Verfassungsgerichte als demokratische Akteure, aaO. (FN 9), S. $308 \mathrm{ff}$.

ZfP 60. Jg. 1/2013 
daher keineswegs ein Zufall, dass das Gericht und seine Judikatur zum Vorbild der nach 1990 geschaffenen Verfassungsgerichte Mittel- und Osteuropas avancierten. ${ }^{60}$

Diese überaus positive Bilanz des Karlsruher Wirkens kann so lange Gültigkeit beanspruchen, wie unter $>$ Demokratie e eine liberale, rechtsstaatliche Demokratie verstanden wird, in der individuelle Grundrechte institutionalisiert sind und vor einem beliebigen Eingriff politischer und gesellschaftlicher Mehrheiten geschützt werden. Verstünde man unter Demokratie in erster Linie die bloße Mehrheitsherrschaft gewählter Volksvertreter (oder auch des Volkes selbst), fiele die Bilanz des Gerichts natürlich etwas anders aus. ${ }^{61}$

Doch unabhängig davon, welchem Demokratiemodell man anhängt: Dass das Bundesverfassungsgericht einen großen Einfluss auf die Politik der Bundesrepublik ausgeübt hat - und nach wie vor ausübt -, wird man zweifellos konstatieren dürfen. ${ }^{62}$ Und zumindest bei einer qualitativen Betrachtung drängt sich der Eindruck auf, dass dieser Einfluss auch im internationalen Vergleich außergewöhnlich stark ist. ${ }^{63}$ Allein mit der institutionellen Ausstattung des Bundesverfassungsgerichts lässt sich dies, wie oben argumentiert worden ist, nicht erklären. Womit aber dann?

Der vergleichsweise große Einfluss des Bundesverfassungsgerichts auf Politik und Gesellschaft hat nicht zuletzt mit dem eigenen Rollenverständnis des Gerichts zu tun. Seine starke institutionelle Ausstattung ist notwendig, aber sicher nicht hinreichend für die starke Rolle des Gerichts im politischen Leben der Bundesrepublik. Hinzutreten muss der Wille des Gerichts (bzw. seiner Richterinnen und Richter), die vorhandenen institutionellen Möglichkeiten auch tatsächlich zu nutzen. Und dieser > Wille< speist sich direkt aus der dem Gericht zugeschriebenen Rolle sowie aus dem Rollenverständnis des Gerichts selbst.

Worin besteht diese Rolle? Vier unterschiedliche Rollenbilder bieten sich an: das Gericht als >Hüter der Verfassung im Sinne Hans Kelsens, ${ }^{64}$ als >konfliktbearbeitender

60 Vgl. Herman Schwartz, The Struggle for Constitutional Justice in Post-Communist Europe, Chicago 2000, S. xi \& 16; Klaus von Beyme, »Modell für neue Demokratien? Die Vorbildrolle des Bundesverfassungsgerichts « in: Robert Chr. Van Ooyen / Martin H. W. Möllers (Hg.), Das Bundesverfassungsgericht im politischen Prozess, Wiesbaden 2006, S. 519-531.

$61 \mathrm{Vgl}$. hierzu ausführlich Sascha Kneip, Verfassungsgerichte als demokratische Akteure, aaO. (FN 9), S. 284-347.

62 Bezieht man die von Christine Landfried herausgearbeiteten Vorwirkungen verfassungsgerichtlicher Rechtsprechung auf das Gesetzgebungsverfahren ebenfalls ein, wird der Einfluss des Gerichts auf die Politik noch deutlicher. Vgl. Christine Landfried, Bundesverfassungsgericht und Gesetzgeber. Wirkungen der Verfassungsrechtsprechung auf parlamentarische Willensbildung und soziale Realität, Baden-Baden 1984.

63 Vergleichende empirisch-quantitative Untersuchungen zum Urteilsoutput von Verfassungsgerichten und ihrem Einfluss auf die Politik und die demokratische Qualität eines Landes liegen derzeit noch nicht vor.

64 Hans Kelsen, »Wer soll Hüter der Verfassung sein?« in: Die Justiz VI (1930/31), S. 576-628. 
Schiedsrichter $<{ }^{65}$ als $>$ Richter und Schlichter ${ }^{66}$ oder gar als $>$ Erzieher $\triangleleft{ }^{67}$ der Bürger, der Exekutive und der Legislative.

Bei der eingangs zitierten 'Zuchtmeisterrolle< des Gerichts schimmert hinsichtlich der öffentlichen Wahrnehmung der 1970er Jahre durchaus eine Art Erziehungsfunktion durch. Das Gericht wurde - zumindest zeitweise und spiegel-typisch überspitzt - als ein Akteur wahrgenommen, der hin und wieder dazu neigte, sich selbst als Praeceptor Germaniae zu begreifen, als (Ober-)Lehrer der Bundesrepublik. Ob dem tatsächlich so war, sei dahingestellt; sicher ist, dass das Gericht heute nicht mehr so gesehen wird. Die verfügbaren Umfragedaten zum Bundesverfassungsgericht deuten vielmehr darauf hin, dass die Bürger es eher als eine Mischung aus gerechtem Richter, fairem Schiedsrichter und unerschrockenem Hüter der Verfassung wahrnehmen. ${ }^{68}$ Auch die Richterinnen und Richter des Bundesverfassungsgerichts selbst können sich in dieser Rollencharakterisierung offenbar mehr oder weniger wiedererkennen. ${ }^{69}$

Aus demokratietheoretischer Perspektive lässt sich das Bundesverfassungsgericht vermutlich am besten als >Hüter der geschriebenen und ungeschriebenen Verfassung beschreiben, und dies vor allem aus zwei Gründen: wegen seiner Konfliktfähigkeit gegenüber der Exekutive und Legislative sowie seiner ausgeprägten Grundrechtsjudikatur. Beides unterscheidet das Bundesverfassungsgericht von manch anderen Verfassungsgerichten der Welt; die Art und Weise, wie es die >Hüterrolle angenommen und ausgestaltet hat, ist auch im internationalen Vergleich außergewöhnlich. Vergleicht man diesbezüglich noch einmal so starke Gerichte wie das Bundesverfassungsgericht, den US-amerikanischen Supreme Court und den österreichischen Verfassungsgerichtshof, fallen gerade in Bezug auf die Konfliktfähigkeit gegenüber der Exekutive und Legislative, aber auch hinsichtlich des Grundrechtsschutzes deutliche Unterschiede auf. Der Supreme Court ist traditionell deutlich zurückhaltender darin, exekutive Machtbefugnisse einzuschränken, und auch die Konfliktbereitschaft gegenüber dem Kongress ist keineswegs so ausgeprägt, wie man gemeinhin vermutet. ${ }^{70}$ Zudem scheint durch das freie Annahme-

65 Vgl. Christian Boulanger, Hüten, richten, gründen: Rollen der Verfassungsgerichte in der Demokratisierung Deutschlands und Ungarns, Dissertation, Freie Universität Berlin 2012.

66 Vgl. Hans-Peter Schneider, »Richter oder Schlichter? Das Bundesverfassungsgericht als Integrationsfaktor« in: Aus Politik und Zeitgeschichte (APuZ) 49, B16 (1999), S. 9-19.

67 So András Sajó in Bezug auf das ungarische Verfassungsgericht, zitiert in Christian Boulanger, Hüten, richten, gründen, aaO. (FN 65), S. 308.

68 Vgl. hierzu auch die Daten bei Hans Vorländer / André Brodocz, »Das Vertrauen in das Bundesverfassungsgericht «, aaO. (FN 16).

69 Vgl. schon die Statusdenkschrift des Gerichts von 1952 sowie die richterlichen Selbstcharakterisierungen bei Uwe Kranenpohl, Hinter dem Schleier des Beratungsgeheimnisses. Der Willensbildungs- und Entscheidungsprozess des Bundesverfassungsgerichts, Wiesbaden 2009, insb. Kapitel 12.

70 Vgl. Winfried Brugger, Grundrechte und Verfassungsgerichtsbarkeit, aaO. (FN 30), S. 16ff. So ist es wohl kein Zufall, dass die genuin amerikanischen >Erfindungen< des judicial self restraint oder die political question doctrine bei uns nie wirklich auf Gegenliebe gestoßen sind. Vgl. hierzu auch Gunnar Folke Schuppert, »Self-restraints der Rechtsprechung. Überlegungen zur Kontrolldichte in der Verfassungs- und Verwaltungsgerichtsbarkeit « in: Deutsches Verwaltungsblatt 103, Nr. 24 (1988), S. 1191-1200. 
verfahren des Supreme Court auch der Grundrechtsschutz letztlich deutlich schwächer ausgeprägt als im bundesdeutschen Fall. ${ }^{71}$

Der österreichische Verfassungsgerichtshof stellt einen interessanten Fall nachholender Entwicklung (auch) nach dem Vorbild des Bundesverfassungsgerichts dar. Er hat erst in den letzten drei Jahrzehnten begonnen, die Verfassung nicht mehr nur als formelle, sondern auch als materielle zu begreifen, deren Grundrechtsgehalte durch die Verfassungsgerichtsbarkeit zu schützen sind. Der Gerichtshof ist damit nicht mehr der vermeintlich unpolitische Schlichter formaler bundesstaatlicher Konflikte, als der er sich lange selbst gesehen hat, sondern er ist langsam - und nach europäischem und bundesdeutschem Vorbild - zu einem Hüter der demokratischen Spielregeln und individuellen Grundrechte avanciert, der heute dem Bundesverfassungsgericht in nahezu keinem Punkt mehr nachsteht. Nicht zuletzt die ausgeprägte Grundrechtsjudikatur des Bundesverfassungsgerichts und seine Rechtsprechung zum Gleichheitssatz führten in Österreich zu einem Umdenken bezüglich der Rolle der Verfassungsgerichtsbarkeit. ${ }^{72}$ Diese Vorbildfunktion übte und übt das Bundesverfassungsgericht auch heute noch für andere Gerichte in der Welt aus, etwa für jene in Ungarn oder Südafrika, um zwei prominente Beispiele zu nennen. Das Karlsruher Gericht ist insofern vielleicht nicht (mehr) der allein herausragende Protagonist einer spezialisierten Verfassungsgerichtsbarkeit - an seinem Einfluss und Erfolg sowie an seiner Vorbildfunktion für andere Gerichte hat sich bislang nichts geändert.

\section{Fazit}

Die institutionelle Ausstattung des Bundesverfassungsgerichts und seine formale Stellung im Staatsgefüge sind heute keineswegs mehr einzigartig. Viele junge und jüngere Verfassungsordnungen in Süd-, Mittel- und Osteuropa, aber auch in Lateinamerika, Asien und Afrika haben erfolgreich die institutionelle Struktur des Bundesverfassungsgerichts kopiert und ihre jeweiligen Verfassungsgerichte mit zumindest ähnlicher formaler Stärke und Unabhängigkeit ausgestattet. Gleichwohl besitzt das Bundesverfassungsgericht eine besondere Machtstellung im Staatsgefüge der Bundesrepublik, die auch im internationalen Vergleich außergewöhnlich ist. Verglichen mit anderen höchsten Gerichten beruht diese starke Stellung zum einen auf einer förderlichen Kombination kluger institutioneller Arrangements, zum anderen aber vor allem auf der hohen empirischen Legitimität des Bundesverfassungsgerichts sowie dem spezifischen Rollenverständnis

71 Dies heißt nicht, dass der Grundrechtsschutz im US-amerikanischen Rechtssystem insgesamt schwächer ausgeprägt wäre. Das diffuse System der Verfassungsgerichtsbarkeit ermöglicht prinzipiell eine ebenso große Kontrolldichte. Lediglich der Schutz durch das Oberste Gericht selbst scheint - angesichts deutlich geringerer Chancen, vom Gericht überhaupt angehört zu werden - aus Sicht des einzelnen Bürgers quantitativ wie qualitativ weniger umfassend zu sein als im bundesdeutschen Fall.

72 Zur Entwicklung des Verfassungsgerichtshofs vgl. z. B. Walter Barfuß, Neue Entwicklungen in der Rechtsprechung des Verfassungsgerichtshofs « in: Österreichische Juristen-Zeitung 44, Nr. 22 (1989), S. 673-681. 
des Gerichts. Die hohe Legitimität transformiert institutionell-theoretische in faktische Macht. Das Rollenverständnis des Gerichts, Hüter der demokratischen Verfassung zu sein, sorgt dafür, dass das Gericht überaus konfliktfähig gegenüber der Exekutive und Legislative auftritt, ohne dabei - neben seiner eigenen Stellung - die Grundrechte der Bürgerinnen und Bürger aus den Augen zu verlieren. Beides ist für die demokratiefördernde Wirkung des Gerichts sowie seine auch international herausragende Stellung von entscheidender Bedeutung.

\section{Zusammenfassung}

Das Bundesverfassungsgericht ist hinsichtlich seiner formalen und faktischen Machtfülle, seiner institutionellen Unabhängigkeit und seiner faktischen Interventionstätigkeit im internationalen Vergleich durchaus herausragend, aber keineswegs einzigartig. Zumindest in formaler Hinsicht haben mittlerweile andere Verfassungsgerichte aufgeschlossen. Was das Bundesverfassungsgericht aber nach wie vor von anderen Gerichten unterscheidet, sind seine hohe empirische Legitimität und sein Rollenverständnis. Während seine hohe Legitimität dafür sorgt, dass aus institutioneller Macht faktische wird, hat das Rollenverständnis als >Hüter der demokratischen Verfassung zu außergewöhnlicher Konfliktfähigkeit einerseits und demokratiefunktionaler Rechtsprechung andererseits beigetragen. Beides macht das Bundesverfassungsgericht immer noch zum herausragenden Vorbild einer >demokratischen $<$ Verfassungsgerichtsbarkeit.

\section{Summary}

In terms of its formal and de facto powers, its institutional independence, and its effective intervention activities, the German Federal Constitutional Court (FCC) is still quite outstanding by international standards, but by no means unique. At least formally, other constitutional courts of the world have caught up to the FCC. But the FCC is still exceptional because of two aspects: its extremely high empirical legitimacy and its selfperception regarding its role in German politics. While the high empirical legitimacy of the court transforms formal into real power, the self description as a $>$ guardian of the democratic constitution< leads to an exceptional ability to deal with conflicts with the political branches and to deliver a democracy-friendly jurisdiction. The FCC remains an outstanding example of a court that is highly functional to democratic governance.

Sascha Kneip, Role and Impact of the German Federal Constitutional Court in Comparative Perspective 